\title{
Resolving the Structure of Ionized Helium in the Intergalactic Medium with the Far Ultraviolet Spectroscopic Explorer
}

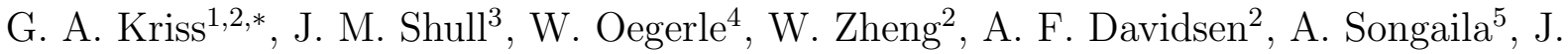 \\ Tumlinson $^{3}$, L. L. Cowie ${ }^{5}$, J.-M. Deharveng ${ }^{6}$, S. D. Friedman ${ }^{2}$, M. L. Giroux ${ }^{3}$, R. F. \\ Green $^{7}$, J. B. Hutchings ${ }^{8}$, E. B. Jenkins ${ }^{9}$, J. W. Kruk ${ }^{2}$, H. W. Moos ${ }^{2}$, D. C. Morton ${ }^{8}$, K. R. \\ Sembach $^{2}$, T. M. Tripp ${ }^{9}$
}

\begin{abstract}
The neutral hydrogen (H I) and the ionized helium (He II) absorption in the spectra of quasars are unique probes of structure in the early universe. We present FarUltraviolet Spectroscopic Explorer observations of the line of sight to the quasar HE23474342 in the 1000-1187 Angstrom band at a resolving power of 15,000. We resolve the He II Ly $\alpha$ absorption as a discrete forest of absorption lines in the redshift range 2.3 to 2.7. About 50 percent of these features have $\mathrm{H}$ I counterparts with column densities $N_{H I}>10^{12.3}$ per square centimeter that account for most of the observed opacity in He II Ly $\alpha$. The He II to H I column density ratio ranges from 1 to $>1000$ with an average of $\sim 80$. Ratios of $<100$ are consistent with photoionization of the absorbing gas by a hard ionizing spectrum resulting from the integrated light of quasars, but ratios of $>100$ in many locations indicate additional contributions from starburst galaxies or heavily filtered quasar radiation. The presence of He II Ly $\alpha$ absorbers with no H I counterparts indicates that structure is present even in low-density regions, consistent with theoretical predictions of structure formation through gravitational instability.
\end{abstract}

\footnotetext{
${ }^{1}$ Space Telescope Science Institute, 3700 San Martin Drive, Baltimore, MD 21218

${ }^{2}$ Center for Astrophysical Sciences, Department of Physics and Astronomy, The Johns Hopkins University, Baltimore, MD 21218-2686

${ }^{3}$ CASA and JILA, Department of Astrophysical and Planetary Sciences, University of Colorado, Campus Box 389, Boulder, CO 80309

${ }^{4}$ Laboratory for Astronomy and Solar Physics, Code 681, NASA/Goddard Space Flight Center, Greenbelt, MD 20771

${ }^{5}$ University of Hawaii, Institute for Astronomy, 2680 Woodlawn Road, Honolulu, HI 96822

${ }^{6}$ Laboratorie d'Astronomie Spatiale, BP 8, 13376 Marseille Cedex 12, France

${ }^{7}$ Kitt Peak National Observatory, National Optical Astronomy Observatories, P. O. Box 26732, 950 North Cherry Ave., Tucson, AZ, 85726-6732

${ }^{8}$ Herzberg Institute of Astrophysics, National Research Council of Canada, Victoria, BC, V8X 4M6, Canada

${ }^{9}$ Princeton University Observatory, Princeton, NJ 08544

* To whom correspondence should be addressed. E-mail: gak@stsci.edu
} 
The intergalactic medium (IGM) is the gaseous reservoir that provides the raw material for the galaxies that dominate our view of the visible universe. By observing distant bright objects such as quasars, we can explore the IGM by examining the absorption features it imprints on the transmitted light. These absorption features trace structure in the universe at epochs intermediate between the earliest density fluctuations seen in the cosmic background radiation and the distribution of galaxies visible today. The distribution of absorption features according to redshift $(z)$ and the column densities of gaseous material in different ions reveal the structure of the IGM and its density and ionization state. From the ionization state of the gaseous species, we can also infer the processes responsible for ionizing the gas, e.g., radiation from quasars in the early universe, or from early bursts of star formation.

The lack of smooth Ly $\alpha$ absorption by $\mathrm{H}$ I in quasar spectra (1) led to the conclusion that any diffusely distributed gas must be too highly ionized to be visible. Discrete absorption features were so plentiful, however, that they were dubbed the "Lyman $\alpha$ forest" (2). Because ionized helium $\left(\mathrm{He}^{+}\right)$is more difficult to ionize than hydrogen (ionization potential of $54.4 \mathrm{eV}$ versus $13.6 \mathrm{eV}$ ) and recombines faster, its abundance in the IGM is expected to be higher. Searches for a diffuse component of the IGM therefore concentrated on absorption from He II Ly $\alpha \lambda 304 \AA$ (3). This absorption was first observed using the Hubble Space Telescope (HST) (4) along the line of sight to the quasar Q0302-003 $(z=3.29)$. The observation showed the IGM to be essentially opaque at redshifts of $z \sim 3$, and it seemed possible that the material in the $\mathrm{H}$ I Ly $\alpha$ forest was sufficient to account for the He II opacity (5). However, because no discrete He II features could be observed, this required several assumptions. Measurement (6) of the $\mathrm{He}^{+}$opacity at lower $z$, using the Hopkins Ultraviolet Telescope (HUT), showed a translucent medium with He II optical depth $\tau \sim 1$ at $z \sim 2.4$. The HUT observations were of insufficient spectral resolution to detect discrete He II absorption features. However, models of the sightline towards the quasar HS1700+64 $(z=2.72)$ required a substantial contribution to the He II opacity from $\mathrm{He}^{+}$that was more smoothly distributed than the known H I Ly $\alpha$ absorbers $(6,7)$ and improved $H S T$ observations of Q0302-003 also required more He II opacity than that predicted by the H I absorption (8). Resolution of these inconsistencies requires observations at higher spectral resolution.

Recent theoretical studies view the highredshift IGM as a tracer of cosmic structure formation by gravitational instability. In such a scenario, diffusely distributed baryonic material (the protons and neutrons that constitute ordinary matter) responds to the gravitational influence of the underlying dark matter. The most overdense regions collapse first to form the earliest galaxies. This leaves the remaining gas as the IGM. Rather than being a uniform medium filling the space between galaxies, the IGM itself should show structur on scales larger than that of individual galaxies. Both numerical calculations (9-16) and analytic theory (17-21) link the evolving structure of the IGM to the $\mathrm{H}$ I and He II absorption visible in the spectra of high redshift quasars.

Given the unsettled observational issues and the need to test the theoretical expectations, we planned Far Ultraviolet Explorer (FUSE) observations of the He II absorption toward the bright (visual magnitude $\mathrm{V}=16.1$ ) $z=2.885$ quasar HE2347-4342 at high spectral resolution. Previous observations of HE2347-4342 at longer ultraviolet (UV) wavelengths showed it to be one of the brightest candidates for such observations (22, 23). As with the other quasars observed with HST, the He II absorption in this object is mostly opaque, but there 
are wavelength intervals of high transmission that suggest we can see the beginnings of the $\mathrm{He}^{+}$re-ionization in the IGM in the redshift range accessible toward this quasar (22). Because He II Ly $\alpha$ at $z=2.885$ is redshifted to $1181 \AA$ and HST is sensitive only down to 1150 $\AA$, which corresponds to $z=2.79$, the $H S T$ data do not cover a large range in redshift. We therefore hoped to trace this re-ionization process over a greater range with FUSE because its short-wavelength sensitivity would let us probe the IGM to much lower redshifts, down to $z \sim 2.0$ in principle (24).

We observed HE2347-4342 with FUSE in two separate campaigns. The first observation, from 17 to 27 August 2000, comprised 351,672 $\mathrm{s}$, of which $192,610 \mathrm{~s}$ was during orbital night (25). The second, running from 11 to 21 October 2000 , accumulated 249,717 s, with 183,630 s during orbital night. For each observing campaign, HE2347-4342 was centered in the 30 arc sec $\times 30$ arc sec apertures. To maintain the optical alignment of the four channels during these campaigns, we offset FUSE every other day to the nearby UV-bright star WD2331-475 and performed routine adjustments to the mirror positions.

At the faint flux levels presented by HE2347$4342\left(F_{\lambda} \sim 3 \times 10^{-15} \mathrm{erg} \mathrm{cm}^{-2} \mathrm{~s}^{-1} \AA^{-1}\right.$ at 1200 $\AA)$, an accurate extraction of a backgroundsubtracted, calibrated source spectrum from the two-dimensional (2D) recorded data required customized processing. Because the $\mathrm{LiF}$ channels have a throughput that is $\sim 3$ times that of the $\mathrm{SiC}$ channels, we discuss only the LiF portions of the spectrum. Because there are spatial variations in the background that affect the extracted flux level in a source as faint as HE2347-4342, we turned off the standard background subtraction in the FUSE data-processing pipeline and extracted a "source + background" spectrum (26). We then manually extracted background spectra from the geometrically recti- fied detector images using regions lying adjacent to the source spectrum. Dead spots and airglow lines from other FUSE apertures were masked out of these background spectra. We computed a linear slope joining the two background regions, and scaled the final result for each of the four detector segments to match the level at the location of the extracted source+background spectrum. We subtracted these background spectra from the pipeline-extracted source+background spectra, and then used the rest of the normal pipeline processes to perform the standard wavelength and flux calibrations. At all steps we propagated a 1- $\sigma$ error array along with the data.

The multiplicity of detectors in FUSE and our two separate observations allowed several checks on our reduction process. At each wavelength we obtained two separate extracted spectra from each observation for a total of four independent spectra. All spectral features noted in our combined spectrum were visible in each independent spectrum. As a check on our flux levels, we computed the scatter among the fluxes from the 1000 to $1080 \AA$ spectra $\left(2.7 \times 10^{-16} \mathrm{erg} \mathrm{cm}^{-2} \mathrm{~s}^{-1} \AA^{-1}\right)$ and the 1100 to $1187 \AA$ spectra $\left(2.1 \times 10^{-16} \mathrm{erg} \mathrm{cm}^{-2} \mathrm{~s}^{-1} \AA^{-1}\right)$. For both, the scatter is higher than our 1- $\sigma$ errors, indicating some residual systematic errors. These errors are $\sim 5$ to $10 \%$ of the extrapolated continuum flux for HE2347-4342, and they are indicative of our overall uncertainty. As a check on our zero levels, we note that the interstellar absorption line C II $\lambda 1036$ is saturated in all FUSE extragalactic spectra. All four extracted spectra containing this line have zero flux at line center to within the 1- $\sigma$ error bars. At wavelengths of $>1150 \AA$, previous $H S T$ observations of HE2347-4342 show several regions where there is little or no flux. Again, our extracted spectra also show no net flux in these regions to within our 1- $\sigma$ errors. Given the consistency among these separate observations, we combined the separate spec- 
tra into a single spectrum with a uniform wavelength scale and $0.05 \AA$ bins (Fig. 1).

Because the FUSE bandpass stops short of wavelengths where the unobscured continuum of HE2347-4342 is visible $(\lambda>1190 \AA)$, we obtained low-resolution HST spectra covering $1150-3200 \AA$ on 21 August and 16 October 2000 to establish the continuum level. These observations each consisted of a 1060 s exposure using the Space Telescope Imaging Spectrograph (STIS) with grating G140L and a 600 s exposure using grating G230L. HE2347-4342 was slightly fainter (by 7\%) in the October observation, but otherwise the spectra were identical. We scaled the October observation up to the August flux levels, and fitted a simple power law, $F_{\lambda}=3.31 \times$ $10^{-15}(\lambda / 1000 \AA)^{-2.40} \mathrm{erg} \mathrm{cm}^{-2} \mathrm{~s}^{-1} \AA^{-1}$, with an extinction correction to spectral regions free of galactic absorption lines. We used a mean galactic extinction curve with ratio of selective to total extinction $R_{V}=3.1$ (27) and a color excess $E(B-V)=0.014$ (28), where $B$ is the blue-band magnitude.

The spectrum and extrapolated continuum (Fig. 1) shows that for $z>2.72(\lambda \gtrsim 1130$ $\AA)$, the IGM is relatively opaque, with occasional narrow windows that are nearly transparent (22, 23). At $z=2.72$, the IGM becomes more transmissive, and the opacity systematically drops at lower $z$ and shorter wavelengths. In this translucent region, it is also apparent that discrete absorption features account for most, if not all, of the opacity. We have resolved the He II absorption into a He II Ly $\alpha$ forest, analogous to the $\mathrm{H}$ I $\operatorname{Ly} \alpha$ forest.

The quantitative character of the evolution in opacity with redshift is consistent with our expectations. The mean opacity over the redshift interval 2.3 to 2.7 is $\tau_{\mathrm{HeII}}=$ $0.91 \pm 0.01$ (29), similar to the sightline towards HS1700+64 (6). We compare the measured opacities to a model (21) of discrete clouds photoionized by the general quasar popula- tion, valid for epochs after He II re-ionization is complete. In the model, ionization fluctuations on scales of $\sim 4000 \mathrm{~km} \mathrm{~s}^{-1}(\sim 15 \AA$, the size of our largest bins) lead to the predicted range of opacities (Fig. 2). For $z<2.72$, where He II reionization is potentially complete, the absolute level of the observed opacity, its trend to lower values at lower redshift, and the fluctuations about the mean are all consistent with this model.

The extrapolated STIS continuum is consistent with the peak fluxes in the FUSE spectrum (Fig. 1). Positive residuals from points above the extrapolated continuum have a Gaussian distribution consistent with the $1 \sigma$ error bars on our data points, indicating that there are absorption-free windows where the IGM is transparent in He II Ly $\alpha$. For models in which the physical density of smoothly distributed He II evolves as $n_{\text {HeII }} \propto(1+z)^{\alpha}$, and assuming a deceleration parameter $\Lambda=0$, and density parameter $\Omega=1$, the opacity in smoothly distributed He II Ly $\alpha$ varies with $z$ as $\tau_{\text {smooth }}(z)=$ $\tau_{\text {smooth }}(z=2.885)[(1+z) /(1+2.885)]^{\alpha-1.5}$ for $z<2.885$ (1). By requiring that the distribution of the ratios of positive residuals to the $1-\sigma$ errors be consistent with a Gaussian of mean 0 and dispersion 1 , we set a $1-\sigma$ upper limit on $\tau_{\text {smooth }}(z=2.885)$ by permitting $\tau_{\text {smooth }}(z=2.885)$, the power-law index, and its normalization to vary freely until $\chi^{2}$ increases by 1 . This yields an upper limit of $\tau_{\text {smooth }}(z=2.885)<0.11$ to 0.12 for $\alpha=0$ to 6 .

Because the FUSE data resolve the He II Ly $\alpha$ forest, we can make a detailed comparison of the individual absorption features to their counterparts in the $\mathrm{H}$ I Ly $\alpha$ forest. To measure the individual He II column densities, we fit the FUSE spectrum using the program SPECFIT (30). Our model incorporates the extrapolated continuum, individual He II absorption lines with Voigt profiles (assuming Doppler widths of $b_{\mathrm{HeII}}=b_{\mathrm{HI}}$ ), and foreground interstellar ab- 
sorption in the Milky Way (31). For each He II line, we permit the column density to vary, but we fix $z$ and the line width $\left(b_{\mathrm{HeII}}=b_{\mathrm{HI}}\right)$ to have the same values as those in the $\mathrm{H}$ I line list from a Keck observation of HE2347-4342 with a resolution of $8 \mathrm{~km} \mathrm{~s}^{-1}$ (32). The $\mathrm{H} \mathrm{I}$ lines identified in the Keck spectrum (184 in total) all have He II counterparts (Fig. 3). Although these lines account for most of the observed opacity in He II Ly $\alpha$, they account for only $\sim 50 \%$ of the observed spectral features. To model the remaining features, we inserted additional lines whose redshifts were permitted to vary freely and whose widths were fixed at $b=27 \mathrm{~km} \mathrm{~s}^{-1}$, the mean of the distribution from the $\mathrm{H}$ I line list. Over the 1000 to $1130 \AA$ wavelength range, 179 additional He II Ly $\alpha$ absorption features were added to our fit. At the 3- $\sigma$ confidence limit, we are sensitive to He II lines with a limiting column density of $\log \mathrm{N}(\mathrm{HeII})>10^{12.8} \mathrm{~cm}^{-2}$. For comparison, there are $72 \mathrm{H} \mathrm{I} \mathrm{Ly} \alpha$ features with $\mathrm{N}_{\mathrm{HI}}<10^{13} \mathrm{~cm}^{-2}$ in the same corresponding range in $z$ in the Keck spectrum.

The added features are consistent with an extrapolation of the number of $\mathrm{H}$ I lines per unit column density of the form $f\left(N_{H I}\right) \propto$ $N_{H I}^{-1.5}(21,33,34)$ down to column densities of $\sim 10^{11} \mathrm{~cm}^{-2}$. This is potentially a substantial amount of material, but, given the powerlaw form of the column density distribution, it does not dominate the total mass in the $\operatorname{Ly} \alpha$ forest (19). Because the physical sizes of the absorbers and the ionization corrections are uncertain, we refrain from estimating the amount of mass that this population of absorbers represents. However, in the context of hydrodynamical models of the IGM $(14,19)$, we note that the low-column-density extension of the Ly $\alpha$ forest contains $\sim 10 \%$ of the baryons at $z=2$ to 3 . Our detection of a large number of He II absorption features with no $\mathrm{H}$ I counterparts is consistent with the predictions of these models.

With measured ratios of He II to $\mathrm{H}$ I col- umn densities, $\eta=\mathrm{N}(\mathrm{HeII}) / \mathrm{N}(\mathrm{HI})$, we can infer the shape of the ionizing spectrum illuminating the absorbing gas. Because our data include both measured values and lower limits (Fig. 4) (for He II Ly $\alpha$ features with no H I Ly $\alpha$ counterparts), we use the Kaplan-Meier estimator for censored data (35) to derive a mean value $\langle\log \eta\rangle=1.89 \pm 0.04$ for the full data set. Models of an IGM photoionized by the integrated light from quasars propagated through the IGM $(21,36)$ predict values of $\eta=30$ to 100 for quasar spectra with spectral indices $\alpha_{q}=1.5$ to $2.1\left(f_{\nu} \propto \nu^{-\alpha_{q}}\right)$. Intrinsic spectral indices for quasars (as measured down to a rest wavelength of $\sim 350 \AA$ ) lie in this range (37). Thus, most of the observed absorption features are consistent with photoionization by quasar radiation. However, about $40 \%$ of the absorbers have $\eta>100$, indicating that localized regions of the IGM are photoionized by softer spectra. This might be heavily filtered quasar radiation (in regions where the re-ionization of He II is not yet complete), or stellar radiation from ongoing star formation (21)

There are fluctuations in $\eta$ on the scale of individual spectral features (Fig. 4) which implies that the ionizing radiation field is not uniform. Local radiation sources (which may be either quasars or star-forming galaxies) probably affect $\eta$ and the local opacity of the IGM. This characteristic is most evident at high $z$ in the low-opacity "windows" first seen in HST spectra of HE2347-4342 (22, 23), but it persists to lower redshift where the IGM is less opaque.

By resolving the He II Ly $\alpha$ absorption in the IGM using FUSE, we are able to see structure in the universe that extends into the lowest density regions. The presence of $\mathrm{He}$ II absorption with no $\mathrm{H}$ I counterparts is consistent with hydrodynamical models that predict density fluctuations due to gravitational instabilities on all scales, from the high density peaks that form galaxies to the distribu- 
tion of gas in low-density voids $(9-16,19,20)$. Observations of He II Ly $\alpha$ absorption is the best method to study these regions, and future observations of additional bright quasars with FUSE should provide essential information on the cosmic variance in the structures we see along different lines of sight.

\section{REFERENCES}

1. J. Gunn and B. Peterson, Astrophys.J. 142, 1633 (1965).

2. R. Lynds, Astrophys.J. 164, L73 (1971).

3. P. Jakobsen, et al., Astrophys.J. 417, 528 (1993).

4. P. Jakobsen, et al., Nature 370, 35 (1994).

5. A. Songaila, E. M. Hu, L. L. Cowie, Nature 375, 124 (1995).

6. A. F. Davidsen, G. A. Kriss, W. Zheng, Nature 380, 47 (1996).

7. W. Zheng, A. F. Davidsen, G. A. Kriss, Astron.J. 115, 391 (1998).

8. S. R. Heap, et al., Astrophys.J. 534, 69 (2000).

9. R. Cen, J. Miralda-Escudé, J. P. Ostriker, M. Rauch, Astrophys.J. 437, L9 (1994).

10. Y. Zhang, P. Anninos, M. L. Norman, Astrophys.J. 453, L57 (1995).

11. Y. Zhang, P. Anninos, M. L. Norman, A. Meiksin, Astrophys.J. 485, 496 (1997).

12. L. Hernquist, N. Katz, D. H. Weinberg, J. Miralda-Escudé, Astrophys.J. 457, L51 (1996).

13. J. Miralda-Escudé, R. Cen, J. P. Ostriker M. Rauch, Astrophys.J 471, 582 (1996).

14. R. A. C. Croft, D. H. Weinberg, N. Katz, L. Hernquist, Astrophys.J. 488, 532 (1997).
15. R. Cen, and J. P. Ostriker, Astrophys.J. 514, 1 (1999).

16. R. Davé, L. Hernquist, N. Katz, D. H. Weinberg, Astrophys.J. 511, 521 (1999).

17. H. Bi, Astrophys.J. 405, 479 (1993).

18. H. Bi, J. Ge, L.-Z. Fang, Astrophys.J. 452, 90 (1995).

19. H. Bi and A. F. Davidsen, Astrophys.J. 479, 523 (1997).

20. L. Hui, N. Y. Gnedin, Y. Zhang, Astrophys.J. 486, 599 (1997).

21. M. A. Fardal, M. L. Giroux, J. M. Shull, Astron.J. 115, 2206 (1998).

22. D. Reimers, et al., Astron.Astrophys. 327, 890 (1997).

23. A. Smette et al. Astrophys.J., in press (available at http://xxx.lanl.gov/abs/astro$\mathrm{ph} / 0012193)$.

24. FUSE covers the wavelength range 912 to $1187 \AA$ with four separate optical channels $(38,39)$. Each channel consists of an off-axis paraboloidal mirror feeding a prime-focus Rowland circle spectrograph. The dispersed light is focused on two, 2D photon-counting microchannel-plate detectors with $\mathrm{KBr}$ photocathodes that record the time, position, and pulse height of each photon event. Each detector is split into two segments with a small gap between them. Two channels use LiF-coated optics to cover the band 1000 to $1187 \AA$; the other two channels use SiC coatings to provide wavelength coverage down to $912 \AA$.

25. Because the count rate in a $50,000 \mathrm{~s}$ observation in June 2000 was lower than the typical background rate, we chose the dates of these observations to maximize the exposure time during orbital night, the part of 
the orbit when FUSE is in Earth's shadow. This minimizes the background contribution due to scattered geocoronal Ly $\alpha$.

26. To obtain the lowest background rate possible, we used only orbital night observations, filtered the high-background "bursts" (39) from the data, and used only events with pulse heights in the range 4 to 16 . This reduces noise due to the low pulseheight events that arise from internal detector background rather than from real photons.

27. J. Cardelli, G. Clayton, J. Mathis, Astrophys.J. 345, 245 (1989).

28. D. J. Schlegel, D. P. Finkbeiner, M. Davis, Astrophys.J. 500, 525 (1998).

29. Although the mean opacity is tightly constrained, we note that there is a large dispersion of 0.9 about this value.

30. G. A. Kriss, in Astronomical Data Analysis Software and Systems III, D. R. Crabtree, R. J. Hanisch, J. Barnes, Eds. (A. S. P. Conf. Series 61, ASP, San Francisco, 1994), p. 437.

31. For the low extinction along this sightline, the interstellar absorption model contributes strong features only for Si II $\lambda 1020$, Ly $\beta \lambda 1025$, C II $\lambda 1036$, O I $\lambda 1039$, Ar I $\lambda \lambda 1048,1066$, and no $\mathrm{H}_{2}$ absorption. Along similar high latitude sight lines observed with FUSE, the column density in $\mathrm{H}_{2}$ is on the order of a few $\times 10^{15} \mathrm{~cm}^{-2}[\mathrm{~J}$. M. Shull et al., Astrophys.J. 538, L73 (2000)]. The average opacity produced by such a column density amounts to $<4 \%$ from $1000-1110 \AA$.

32. A. Songaila, Astron.J. 115, 2184 (1998).

33. W. H. Press and G. B. Rybicki,Astrophys.J. 418, 585 (1993).

34. E. M. Hu, T.-S. Kim, L. L. Cowie, A. Songaila, M. Rauch, Astron.J. 110, 1526 (1995).
35. E. D. Feigelson, and P. I. Nelson, Astrophys.J. 293, 192 (1985).

36. P. Madau, F. Haardt, M. J. Rees, Astrophys.J. 514, 648 (1999).

37. W. Zheng, G. A. Kriss, R. C. Telfer, J. P. Grimes, A. F. Davidsen, Astrophys.J. 475, 469 (1997).

38. H. W. Moos, et al., Astrophys.J. 538, L1 (2000).

39. D. Sahnow, et al., Astrophys.J. 538, L7 (2000).

40. We sadly report that Arthur F. Davidsen, one of our key team members and a pioneer in ultraviolet observations of the intergalactic medium, passed away on July 19, 2001. This work is based on data obtained for the Guaranteed Time Team by the NASACNES-CSA FUSE mission operated by the Johns Hopkins University. Financial support to U. S. participants has been provided by NASA contract NAS5-32985, and by the NASA LTSA grant NAG5-7262 to the University of Colorado. A portion of this work is based on observations with the NASA/ESA Hubble Space Telescope, obtained at the Space Telescope Science Institute, which is operated by the Association of Universities for Research in Astronomy, Inc., under NASA contract NAS5-26555. These observations are associated with proposal ID 8875. We thank B. Roberts for his efforts in planning and scheduling the successful FUSE observations, and J. Kim for her help in analyzing the STIS data.

This 2-column preprint was prepared with the AAS LATEX macros v5.0. 


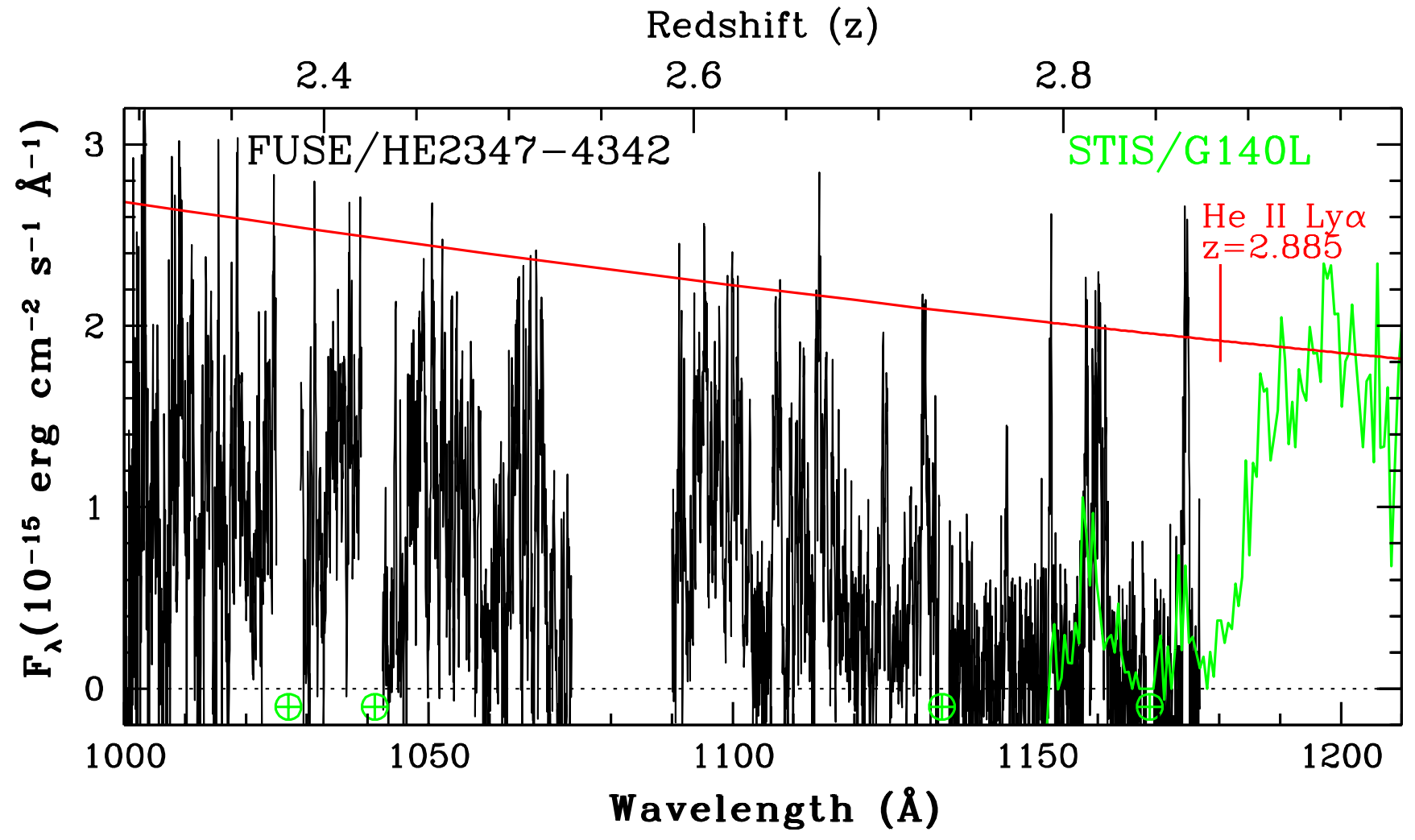

Fig. 1. - FUSE spectrum of HE2347-4342 (binned to $0.05 \AA$ pixels) is shown in black. Bins at the peak fluxes have a signal-to-noise ratio of $\sim 7$. A portion of the contemporaneous STIS spectrum is shown in green. The red line is the extrapolation of the power law plus extinction of $\mathrm{E}(\mathrm{B}-\mathrm{V})=0.014$ fitted to the STIS spectrum. The position of He II Ly $\alpha$ at the redshift of HE2347-4342 is marked. Gaps in the FUSE spectrum due to excised terrestrial airglow lines are marked with green $\oplus$. The broad gap from 1072 to $1089 \AA$ is due to gaps between the FUSE detector segments. 


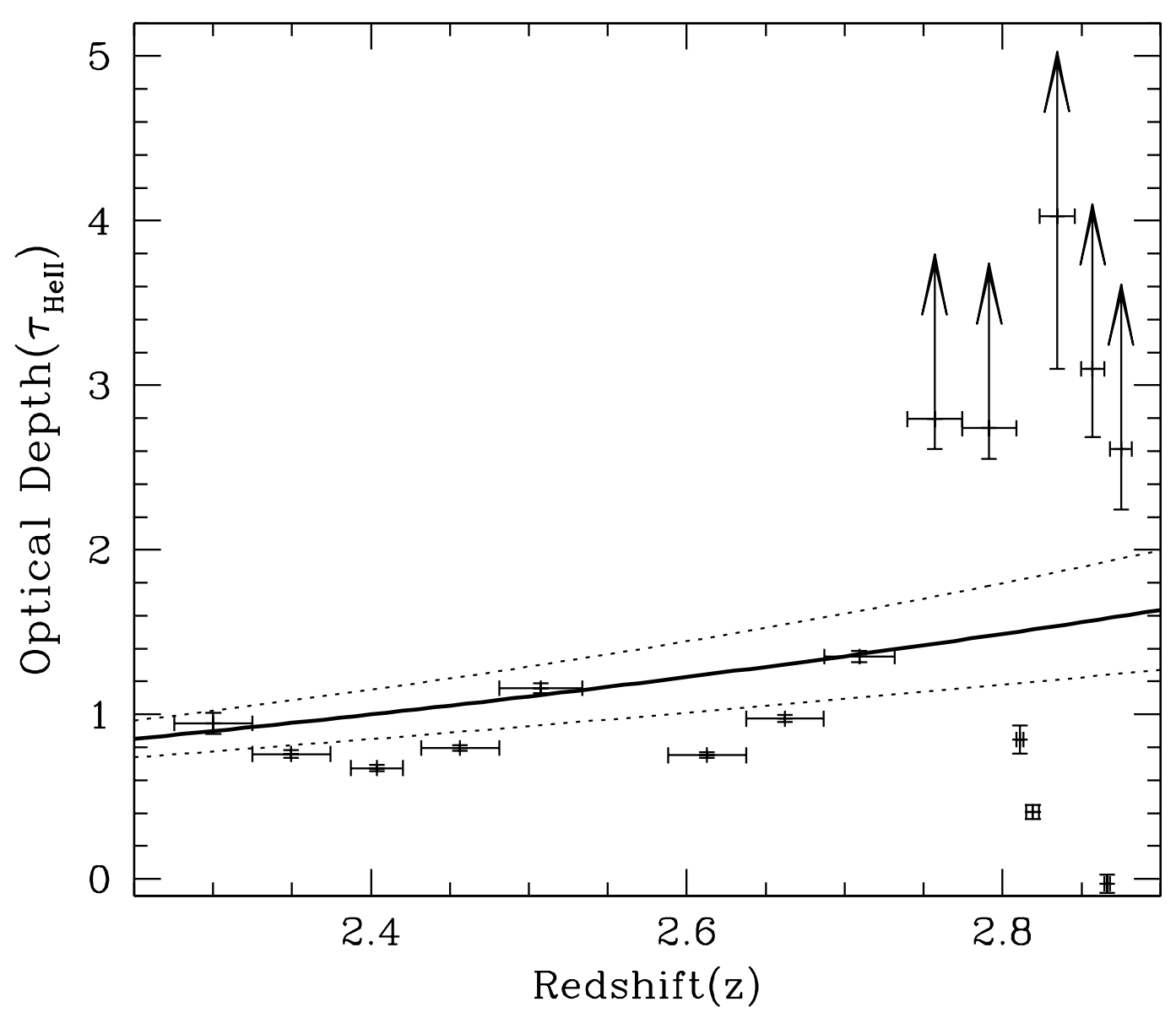

Fig. 2.- The He II opacity $\tau_{H e I I}$ in coarse 5 to $15 \AA$ bins is shown as a function of redshift. The solid curve is the theoretical prediction for the opacity assuming line blanketing only (21). The dashed curves indicate the $1 \sigma$ extent of variations in the opacity anticipated due to ionization fluctuations. At redshifts below $z=2.72$, where the reionization of He II in the IGM appears to be complete, the model matches well the observed opacity, its trend to lower values at lower redshift, and the fluctuations about the mean. 


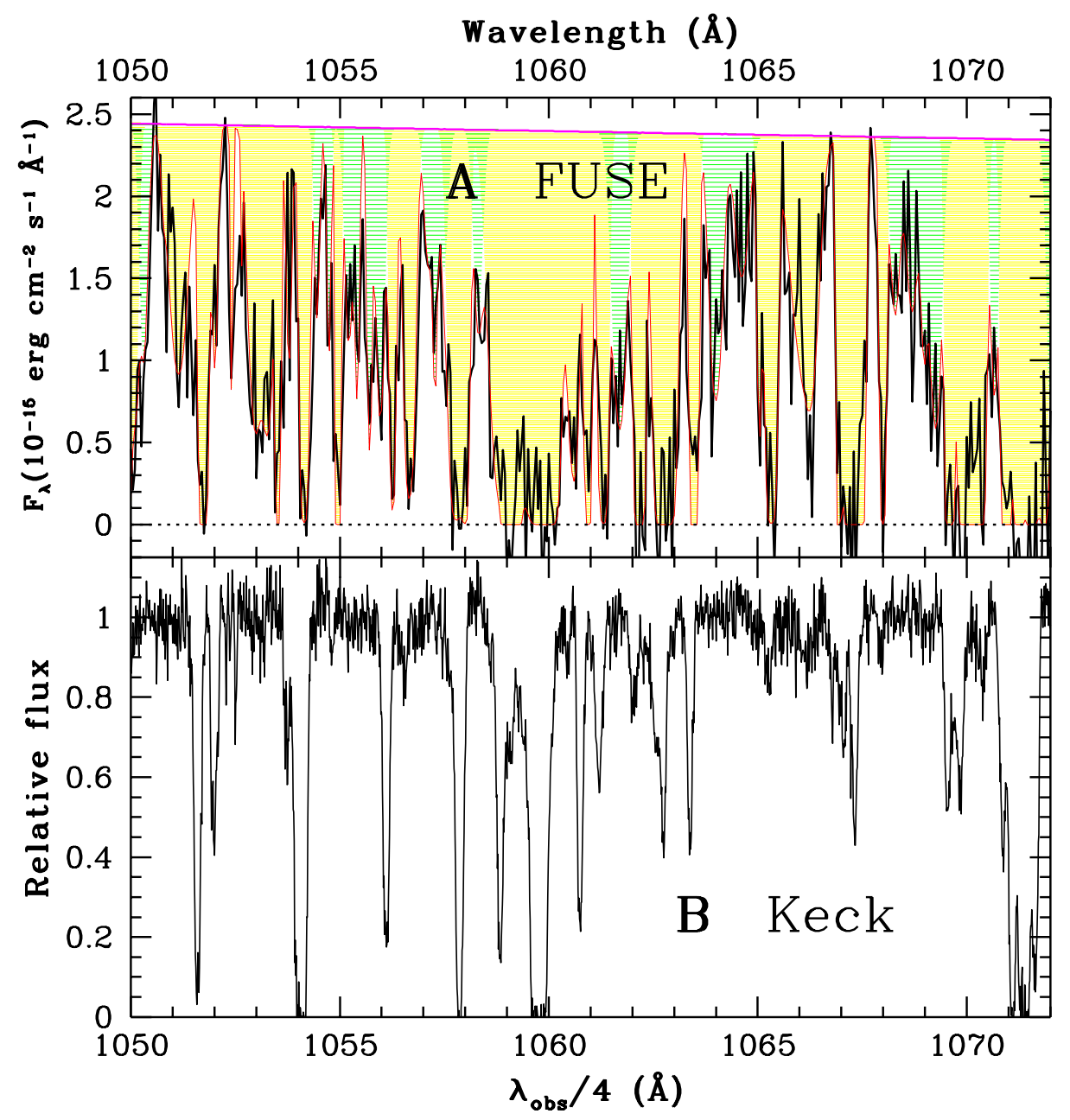

Fig. 3.- (A) The black line is a portion of the FUSE spectrum of HE2347-4342. The smooth red curve across the top is the extrapolated continuum. The magenta line overlaying the spectral data in black is the model described in the text. The area shaded in yellow shows the fraction of the He II opacity due to absorption features that correspond to H I absorption lines identified in the Keck spectrum (32). The area shaded in green shows the fraction of the opacity due to additional He II absorption features that have no H I counterparts in the Keck spectrum. (B) The normalized Keck spectrum (32). Note the direct correspondence between the wavelengths of H I lines in the Keck spectrum and He II absorption features in the FUSE spectrum. 


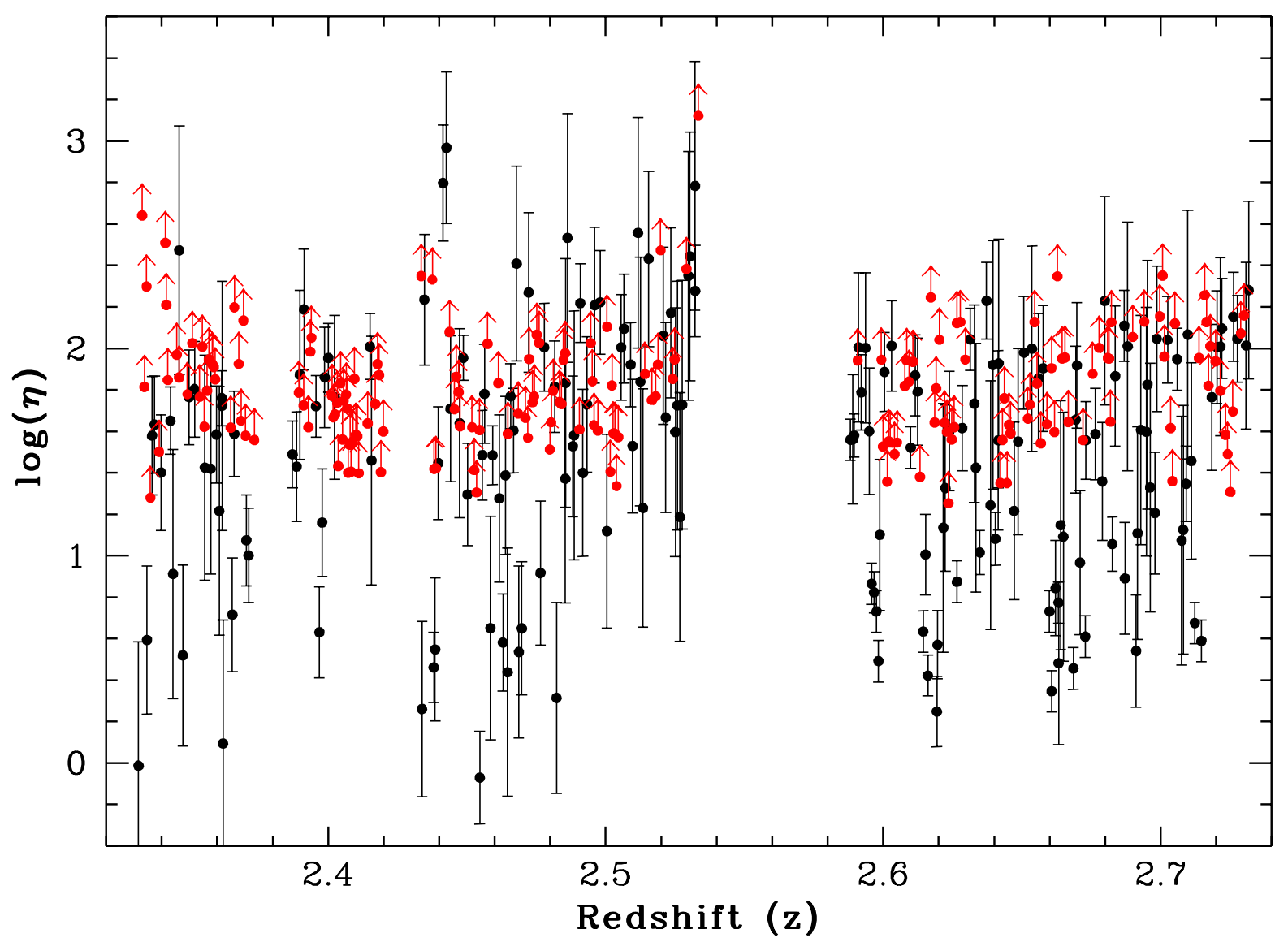

Fig. 4. - The logarithm of the He II to H I column density ratio $\eta=\mathrm{N}(\mathrm{HeII}) / \mathrm{N}(\mathrm{HI})$ versus redshift for measured absorption features in the FUSE spectrum. The black points with error bars have measured column densities for both HeII (from FUSE) and H I (from Keck). The red points are lower limits computed using the He II column density measured from the FUSE spectrum and an upper limit of $10^{12.3} \mathrm{~cm}^{-2}$ for $\mathrm{H}$ I absorption lines in the Keck spectrum. A Spearman rank correlation coefficient of 0.05 indicates that there is no significant trend with redshift. The mean value, $\langle\log \eta\rangle=1.89$, is consistent with photoionization of the IGM by integrated quasar light (21, 36). However, one can see significant fluctuations in $\eta$ that indicate that the ionizing radiation field is not uniform. Values of $\eta>100$ indicate regions photoionized by either heavily filtered quasar radiation or bursts of star formation. 